\title{
EGFR NP_005219.2:p.V769_D770insMASVD
}

National Cancer Institute

\section{Source}

National Cancer Institute. EGFR NP 005219.2:p.V769 D770insMASVD. NCI Thesaurus. Code C98632.

An insertion of the amino acid sequence methionine-alanine-serine-valine-aspartic acid between the valine at position 769 and the aspartic acid at position 770 of the epidermal growth factor receptor protein. 